\section{Combined stents for the treatment of large gastric fistulas or stenosis after sleeve gastrectomy}

Gastric fistulas after sleeve gastrectomy may require placement of a prosthesis such as a covered stent [1-3] or doublepigtail stent (DPS) [4]. In our experience, use of a DPS is associated with a shorter period of hospitalization, lower pain levels, and shorter treatment duration than when a covered stent is used [4]. We present the results for combined covered stent and DPS placement as treatment for large-diameter gastric fistula or gastric stenosis associated with a gastric fistula in four patients. The patients had a $>2-\mathrm{cm}$ large-diameter gastric fistula $(n=2)$ or gastric stenosis associated with a gastric fistula ( $n=2$ ) after sleeve gastrectomy.
The edges of the gastric fistula were visualized with the injection of contrast agent ( Fig.1a,b). A covered stent was implanted to cover the gastric fistula and to expand an associated gastric stricture. With radiological guidance, the endoscope was placed in front of the orifice of the gastric fistula allowing the placement of two DPSs. Using a transprosthetic approach, the two DPSs were placed in order to drain the gastric fistula internally ( $\bullet$ Fig. 1 c-e) and attach the covered stent when a gastric stricture was not present ( $\bullet$ Video 1 ). There was no morbidity related to the procedure. The median number of endoscopic procedures per patient was 3 (range $3-4$ ), and the median number of procedures at the time of covered stent and DPS placement was 1 (range $1-1$ ). The stent migration rate and endoscopic treatment failure rate were $0 \%$. The median total time to complete gastric fistula closure was 57 days (range 54-100 days) and the median time between covered stent placement and gastric fistula closure was 27 days (range 26-28 days).

Use of a covered stent enables us to treat a large-diameter gastric fistula or gastric stenosis with concomitant gastric fistula in some cases, whereas the use of a DPS allows simultaneous drainage of the gastric fistula internally. This combined pro-

\section{Video 1}

New endoscopic technique for treatment of a large-diameter gastric fistula after sleeve gastrectomy.

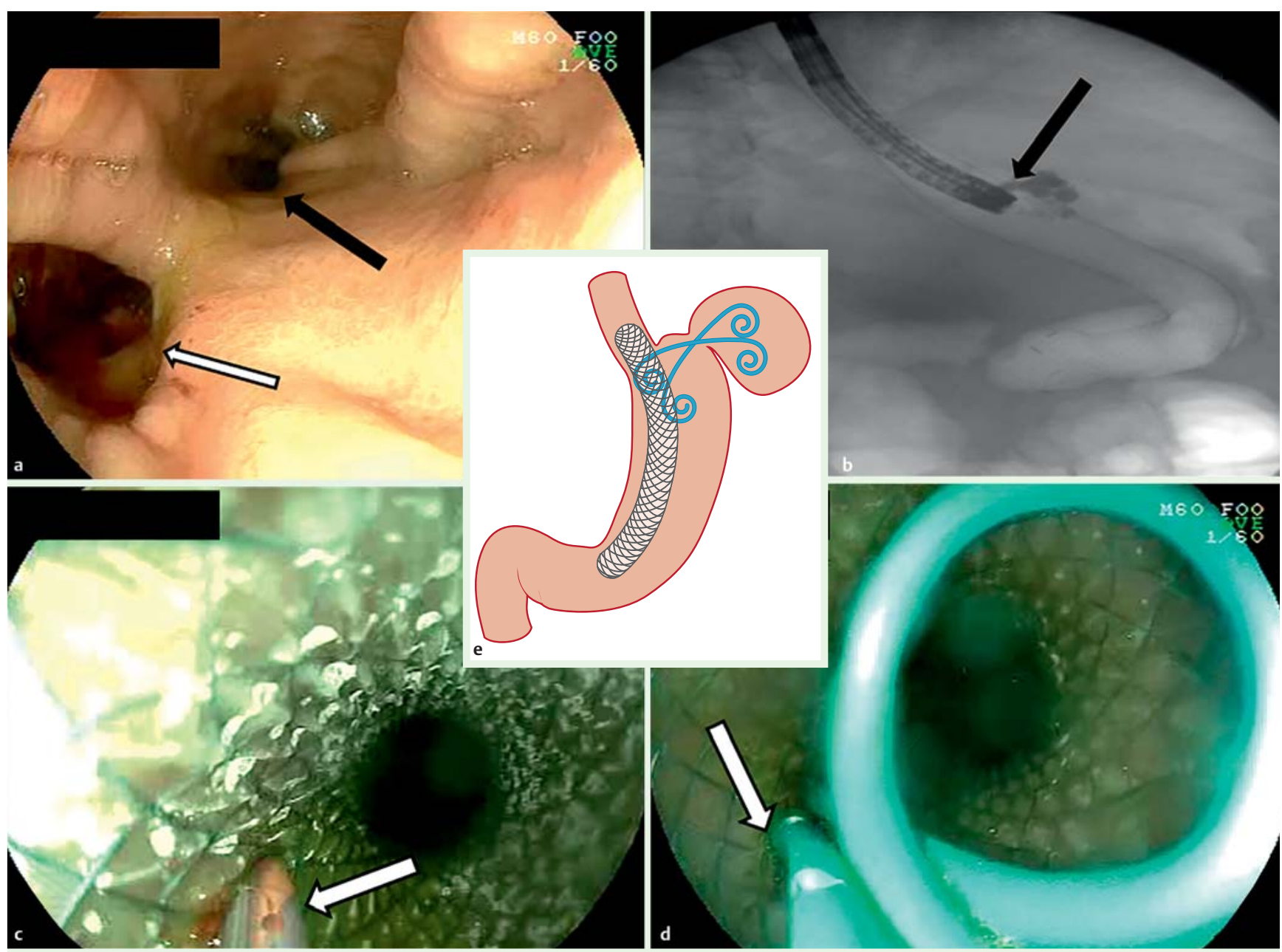

Fig. 1 Combined covered stent and double pigtail stent (DPS) endoscopic procedure as treatment for a large-diameter gastric fistula or gastric stenosis associated with a gastric fistula. a Endoscopy showing a large-diameter gastric fistula (white arrow) next to the gastric lumen (black arrow). b Positioning of the endoscope in front of the gastric fistula (black arrow) after placement of a covered stent with radiological guidance. c Catheterization of the gastric fistula (white arrow). d Placement of two DPSs (white arrow) by a transprosthetic approach. e Frontal pattern of a combined covered stent and DPS placement via a transprosthetic approach. 
cedure avoided the need for additional endoscopies. The absence of prosthesis migration might have been because of gastric stenosis and better fixation of the covered stent when a DPS was placed via a transprosthetic approach. Furthermore, the median time to complete gastric fistula closure was 57 days, mainly because of the short median time interval between covered stent placement and gastric fistula closure (27 days), and the absence of endoscopic treatment failure that would have required subsequent endoscopic procedures then revisional surgery.

These encouraging results should be confirmed in a prospective study with larger sample size to compare its applicability and results for the treatment of large gastric fistulas or gastric strictures with concomitant gastric fistulas.

Endoscopy_UCTN_Code_TTT_1AO_2AZ

Competing interests: None

\section{Lionel Rebibo ${ }^{1}$, Mathurin Fumery ${ }^{2}$, Samy Hakim², Franck Brazier², Abden- naceur Dhahri ${ }^{1}$, Jean-Marc Regimbeau ${ }^{1}$}

${ }^{1}$ Department of Digestive Surgery, Amiens University Medical Center and Jules Verne University of Picardie, Amiens, France

2 Department of Gastroenterology, Amiens University Medical Center and Jules Verne University of Picardie, Amiens, France

\section{References}

1 Sakran N, Goitein D, Raziel A et al. Gastric leaks after sleeve gastrectomy: a multicenter experience with 2,834 patients. Surg Endosc 2013; 27: 240-245

2 Simon F, Siciliano I, Gillet A et al. Gastric leak after laparoscopic sleeve gastrectomy: early covered self-expandable stent reduces healing time. Obes Surg 2013; 23: 687-692

3 Moszkowicz D, Arienzo R, Khettab I et al. Sleeve gastrectomy severe complications: is it always a reasonable surgical option? Obes Surg 2013; 23: 676-686

4 Pequignot $A$, Fuks $D$, Verhaeghe $P$ et al. Is there a place for pigtail drains in the management of gastric leaks after laparoscopic sleeve gastrectomy? Obes Surg 2012; 22: $712-720$
Bibliography

Dol http://dx.doi.org/

10.1055/s-0034-1390711

Endoscopy 2015; 47: E59-E60

(c) Georg Thieme Verlag KG

Stuttgart · New York

ISSN 0013-726X

\section{Corresponding author}

Jean-Marc Regimbeau, MD, PhD

Service de chirurgie digestive

Hôpital Nord

CHU d'Amiens

Place Victor Pauchet

F-80054 Amiens cedex 01

France

Fax: +33-3-22668680

regimbeau.jean-marc@chu-amiens.fr 